\title{
An unusual case of arteriovenous fistula- related venous hypertension: sonographic detection of a culprit perforating vein with movie and compact review
}

\author{
Tadashi Kamata ${ }^{1 *}$, Kojiro Yamamoto ${ }^{1}$, Motohiro Okamura² and Noriyuki lehara ${ }^{1}$
}

\begin{abstract}
Background: Venous hypertension is one of the critical complications of arteriovenous fistula (AV fistula). Here, we report an unusual variation of venous hypertension which was caused by retrograde outflow through a perforating vein of the elbow.

Case presentation: A 79-year-old man with diabetic nephropathy had an AV fistula created at his left proximal forearm 2 years before referral. Shortly after the creation of the AV fistula, he developed swelling of the left hand and forearm. Six months prior to the referral, persistent pain of the left hand developed, and he visited the nephrology unit. An anastomosis of the AV fistula was located $5 \mathrm{~cm}$ distal to the elbow. Inspection, palpation, and auscultation did not suggest outflow stenosis. Ultrasound showed mature upper arm venous outflow without stenosis. His proximal radial artery had been anastomosed side-to-end to a nearby proximal forearm superficial vein. Color Doppler analysis revealed a retrograde outflow through an antecubital perforating vein, which drained into the deep portion of the forearm and then disappeared. Superficial veins of the left forearm had been exhausted due to a previous attempt to create a wrist AV fistula. Given the above, it was suspected that the unusual retrograde outflow through the perforating vein caused venous hypertension by interfering with the venous return of the forearm, which had been dependent on deep veins. The patient subsequently underwent ligation of the perforating vein. The day after the operation, the pain disappeared and swelling improved. The dialysis treatments were continued without problems.
\end{abstract}

Conclusions: Retrograde outflow through a perforating vein can be a cause of venous hypertension in a patient with an AV fistula created using the proximal radial artery. Close sonographic examination of antecubital vessels should be done if a practitioner encounters unilateral whole forearm edema without apparent proximal outflow stenosis.

Keywords: Arteriovenous fistula, Venous hypertension, Perforating vein, Deep communicating vein, Vena mediana cubiti profunda, Diagnosis, Point-of-care ultrasound

\section{Background}

Venous hypertension is one of the critical complications of arteriovenous fistula (AV fistula), which not only interferes with the continuation of adequate dialysis therapy but also degrades the patients' quality of life by affecting the upper arm function or causing pain (Table 1). Generally, venous hypertension is determined by the balance of the degree of outflow stenosis and fistula flow volume [1] and the degree of collateral outflow development. However, a type of venous hypertension that is localized to the whole forearm occurs only under multiple overlapping conditions [2]. Here, we report an unusual variation of the venous hypertension caused by retrograde outflow through a perforating vein of the elbow.

\section{Case presentation}

A 79-year-old man with diabetic nephropathy had an AV fistula created at his left proximal forearm 2 years
1'Department of Nephrology, Kyoto City Hospital, 1-2, Higashi-takada-cho,

Mibu, Nakagyo-ku, Kyoto 604-8845, Japan

Full list of author information is available at the end of the article 
Table 1 Symptoms of AV fistula-related venous hypertension

- $\quad$ Edema of the upper limb
- $\quad$ A decreased range of upper limb joint motion
- $\quad$ Ulceration, pigmentation, and dermatosclerosis of the upper limb
- $\quad$ Superficial venous dilatation over the chest and shoulder ${ }^{a}$
- $\quad$ Headache $^{b}$
- $\quad$ Nasal bleeding $^{\text {b }}$

an the case of axillary, subclavian, or brachiocephalic vein stenosis

${ }^{\mathrm{b}}$ In the case of brachiocephalic vein or superior vena cava stenosis

before referral. One year later, he was started on regular dialysis. Shortly after the creation of the AV fistula, he developed swelling of the left hand and forearm. Six months prior to referral, persistent pain of the left hand developed and he was referred from a dialysis clinic to the nephrology unit because of concerns over the gradual aggravation of the symptoms. His blood pressure was $128 / 57 \mathrm{mmHg}$, pulse was 88 beats/min and regular, and body temperature was $36.3{ }^{\circ} \mathrm{C}$. Physical examination of his chest and abdomen revealed no abnormality. His past medical history was significant for hypertension and chronic obstructive pulmonary disease. He complained of continuous pain of the dorsum of his left hand. On inspection, marked swelling of his left forearm, hand, and fingers was noted (Fig. 1a). An anastomosis of the AV fistula was located $5 \mathrm{~cm}$ distal to the elbow. No stenotic sound was heard along the arm or around the shoulder. A continuous thrill was felt at the anastomosis. Neither swelling of his upper arm nor dilatation of the

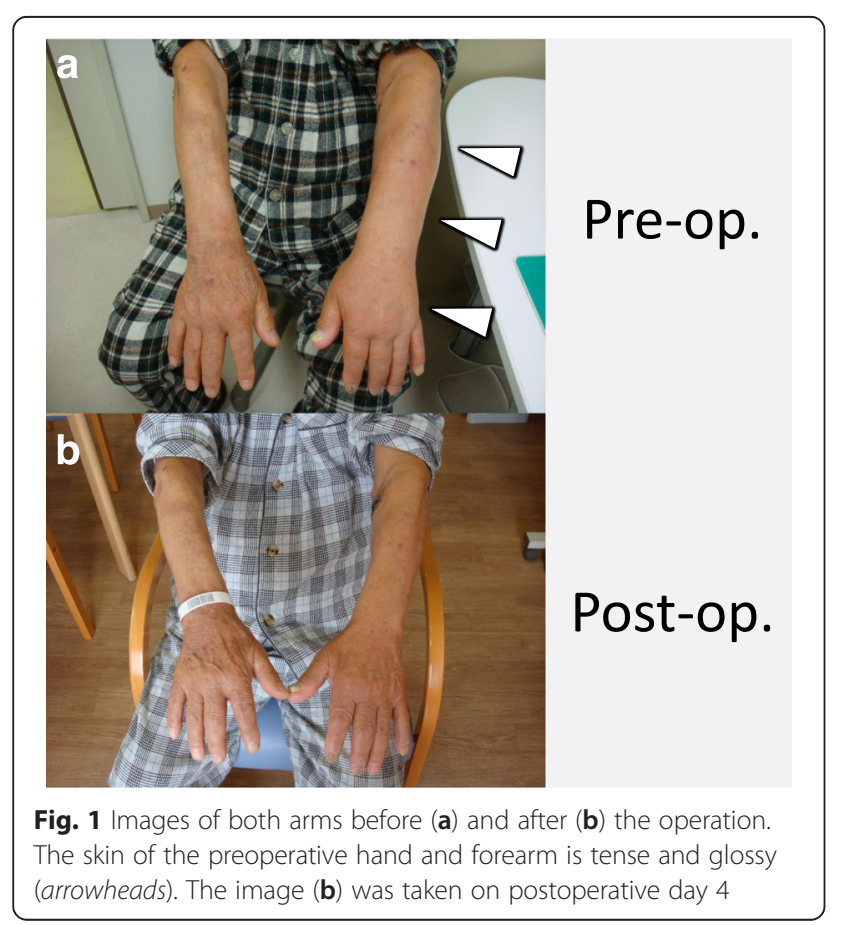

superficial veins around his chest and shoulder was noted. There was neither cold sensation or pallor suggesting arterial steal, nor heat or redness suggesting infection. His dry weight seemed to be appropriate because he had neither leg edema nor uncontrolled hypertension, and his post-dialytic cardiothoracic ratio and human atrial natriuretic peptide level were $49 \%$ and $43.3 \mathrm{pg} / \mathrm{mL}$, respectively.

Ultrasound examination on the day of referral showed a left brachial arterial flow rate of $860 \mathrm{~mL} / \mathrm{min}$ and mature upper arm basilic venous main outflow without stenosis. His proximal radial artery had been anastomosed side-to-end to a nearby proximal forearm superficial vein (i.e., median antebrachial vein). When the ultrasound probe was placed around his left antecubital fossa without a tourniquet, color Doppler analysis revealed retrograde outflow through a perforating vein, which drained into the deep portion of the forearm and then disappeared (Fig. 2a; an additional movie file shows this in more detail [see Additional file 1]). The patient denied a history of central venous catheter use. Supraclavicular ultrasound scanning [3] did not show stenosis of his left subclavian vein. Computed tomography (CT) was performed for conformation, which did not provide additional information (Fig. 3). Superficial veins of the left forearm had been exhausted due to a previous attempt to create a wrist AV fistula. Given the above, it was suspected that the unusual retrograde outflow through the perforating vein caused venous hypertension by interfering with the venous return of the forearm. The patient subsequently underwent ligation of the perforating vein. Five hours after the operation, wrinkling of his left hand skin developed. The day after the operation, the pain of the hand disappeared. The swelling of his left hand and arm also improved, and the difference between the circumferences of the bilateral forearms reduced from 4 to $1.5 \mathrm{~cm}$ after the operation (Fig. 1a, b). Disappearance of the retrograde flow was confirmed by ultrasound (Fig. 2b). His dry weight was not altered in the perioperative period, and the dialysis treatments were continued without problems.

\section{Discussion}

An antecubital perforating vein (also known as a deep communicating vein or vena mediana cubiti profunda) is a connection between the superficial veins such as the cephalic, basilic, and median antebrachial veins, and the deep veins, known as the vena comitans of forearm arteries. The perforating vein is sometimes utilized for proximal forearm fistula for maintenance dialysis $[4,5]$. In spite of its usefulness in maintaining a patent forearm fistula in the case of poor upper arm superficial veins [6], ligation of the perforating vein is recommended when it is not used for anastomosis in the case of an 


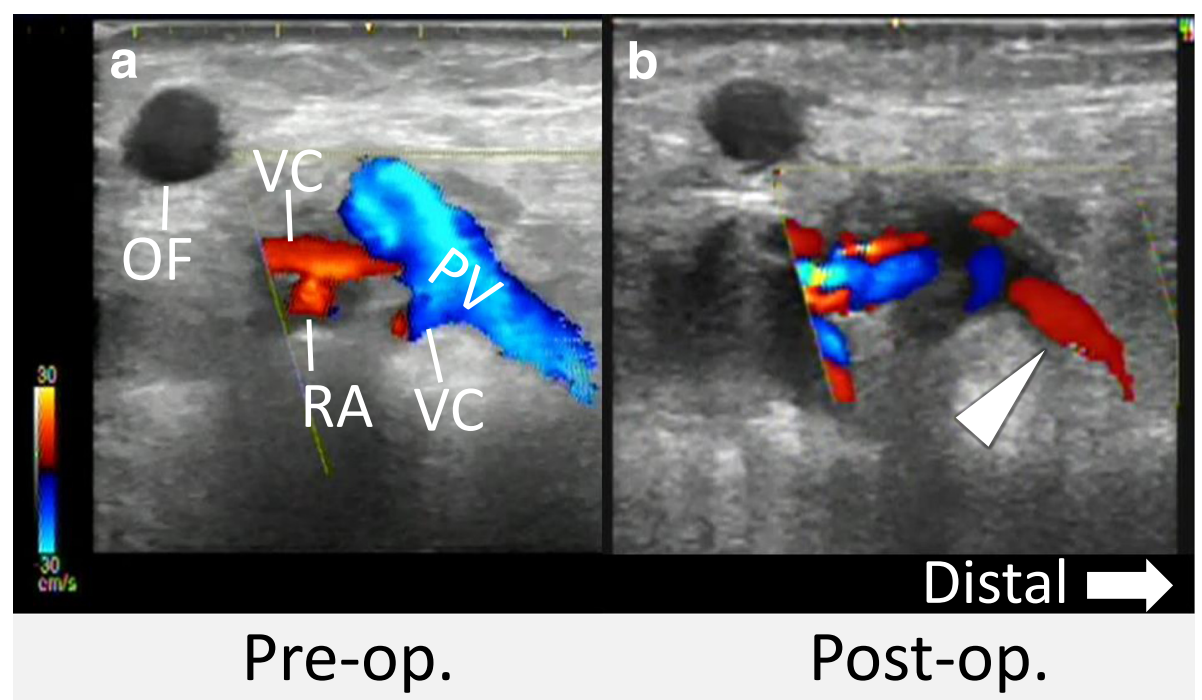

Fig. 2 Longitudinal color Doppler ultrasound views of the perforating vein before (a) and after (b) the operation. a The color of the perforating vein shows that the flow is directed distally and deeply beyond the junction with the venae comitantes of the radial artery. Its flow signal disappears at the deep portion of the forearm. $\mathbf{b}$ Postoperative ultrasound showed antegrade flow through the distal perforating vein (arrowhead). The velocity scale is set to $30 \mathrm{~cm} / \mathrm{s}$. The ultrasound beam is angled to the right side of the screen. OF outflow to the upper arm, $P V$ perforating vein, $R A$ radial artery, VC vena comitans of radial artery

antecubital fistula $[7,8]$. This is due to the concern that flow through the perforating vein may affect maturation by diverting blood to deeper structures. While the importance of a perforating vein is well-known, as described above, what is not mentioned in the literature to

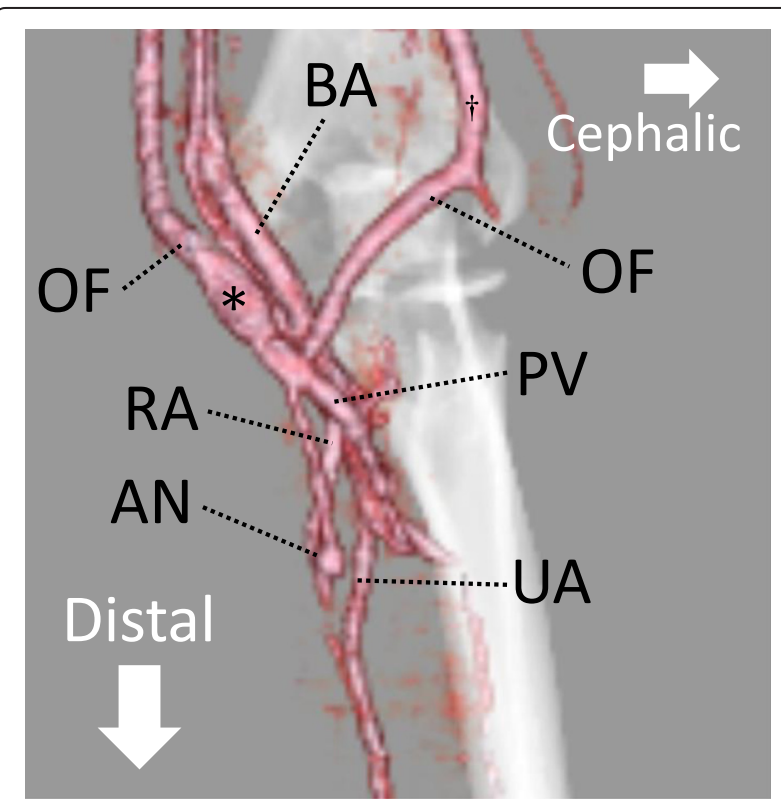

Fig. 3 A preoperative 3D-CT image of the left antecubital veins and arteries. *Cannulation site for an inlet needle, tcannulation site for a return needle. BA brachial artery, AN anastomosis site, UA ulnar artery. The remaining abbreviations are the same as in Fig. 2 our knowledge is forearm venous hypertension due to a perforating vein. In fact, we could not find a similar case in Pubmed, Google Scholar, or the database of Japan Medical Abstracts Society. The pain in our case was not thought to be caused by arterial steal, because the symptoms were not typical of steal and the brachial arterial flow rate was virtually unchanged after the operation notwithstanding the improvement of the symptoms (Table 2). Although the constant pain rapidly disappeared, the swelling was not completely improved, which may reflect damage to the lymphatic system due to the prolonged course [9].

Typically, whole forearm venous hypertension is caused by a proximal forearm side-to-side fistula with antecubital or distal upper arm outflow stenosis [10,11]. Another example is the proximal venous outflow stenosis of a looped arteriovenous graft which connects a brachial artery side-to-side to an elbow basilic vein [2] Neither of those, however, was the case in our patient. There may be four mechanisms to account for the cause of the unusual venous hypertension via the perforating

Table 2 Sonographic blood flow parameters of the left brachial artery before and after the operation

\begin{tabular}{lll}
\hline & Pre-op. & Post-op. \\
\hline Flow volume* $(\mathrm{mL} / \mathrm{min})$ & 860 & 905 \\
Resistive index & 0.51 & 0.53 \\
Pulsatility index & 0.72 & 0.80 \\
\hline
\end{tabular}

Values are expressed as the median of three consecutive measurements ${ }^{*}[$ time-averaged mean velocity $] \times \pi[\text { vessel radius }]^{2}$ 

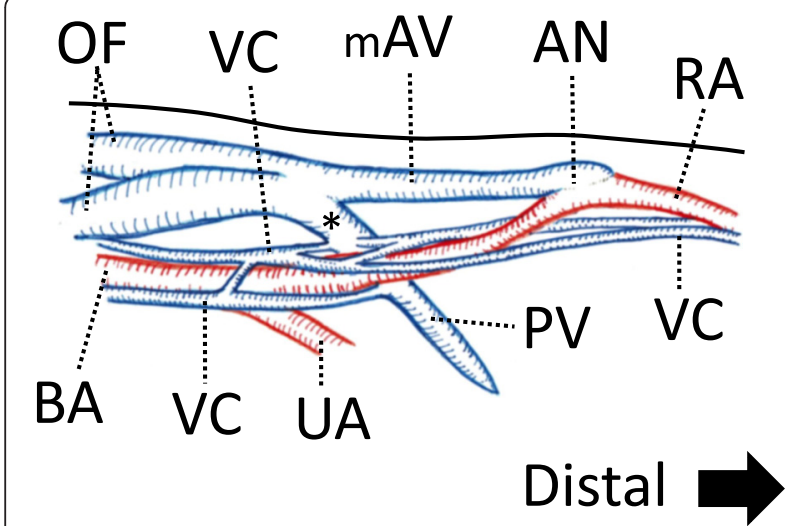

Fig. 4 A schematic diagram of the antecubital vessels based on the ultrasound findings. $m A V$ median antebrachial vein. * Site of ligation. The remaining abbreviations are the same as on Figs. 2 and 3

vein. Firstly, venous return from the forearm was considered to be exclusively dependent on deep arm veins due to the exhaustion of superficial veins. Indeed, we could not find good-quality forearm superficial veins visually, by palpation, or by ultrasonography. Secondly, a venous branch that led to retrograde direct flow to the deep portion (i.e., forearm muscles) existed. In the extensive anatomical study using cadavers done by Berge et al., via a perforating vein, median antebrachial veins connected mainly to a radial vena comitans and with a small number to muscles [12]. Thus, the specific structure of the venous bed in our case may have contributed to the complication (Fig. 4). Thirdly, due to the physical proximity of the perforating vein to the arteriovenous anastomosis, relatively abundant outflow was draining into the perforating vein, although his left brachial arterial flow rate was not extremely high (approximately $900 \mathrm{~mL} / \mathrm{min}$ ). In fact, the distance between the arteriovenous anastomosis and junction of the perforating vein and superficial outflow was approximately $3 \mathrm{~cm}$, between which there were no intervening venous branches. Lastly, there might have been failure of the venae comitantes to dilate as drainage tracts for some reason, such as insufficient resistance [13]. The above-described mechanisms may have synergistically contributed to high-volume retrograde flow being directed distally and deeply, resulting in increased deep soft tissue pressure which interfered with the forearm venous return via the deep veins.

In our case, the findings of ultrasonography were the key to the prompt diagnosis. In addition to exhausted forearm superficial veins, the combination of two sonographic findings may be pivotal to detect the unusual cause of the venous hypertension: (1) retrograde outflow through the perforating vein, which extends deeply beyond the junction with venae comitantes of the radial artery and (2) disappearance of the flow signal at a deep portion of the forearm, which suggests the lack of an effective drainage tract. The combination of these ultrasound findings may be specific because, among 18 hemodialysis patients without perforating vein ligation in our dialysis unit, none of them showed forearm edema or the combination of those sonographic findings (unpublished data). Proof of simple retrograde outflow through a perforating vein alone is not enough because two-thirds of those patients had it. This is not surprising because the flow of a perforating vein can easily be retrograde as it is usually devoid of valves [6]. However, the sensitivity of the sonographic sign is unclear as this is the only case we encountered. The accumulation of further reports is required. Unless the practitioner is familiar with venous hypertension due to a perforating vein, the symptoms may be mistaken for infection or allowed to persist until they become entirely irreversible.

Unilateral upper limb edema on the side of a fistula

Check for inappropriate dry weight, excessive fistula flow, signs of infection

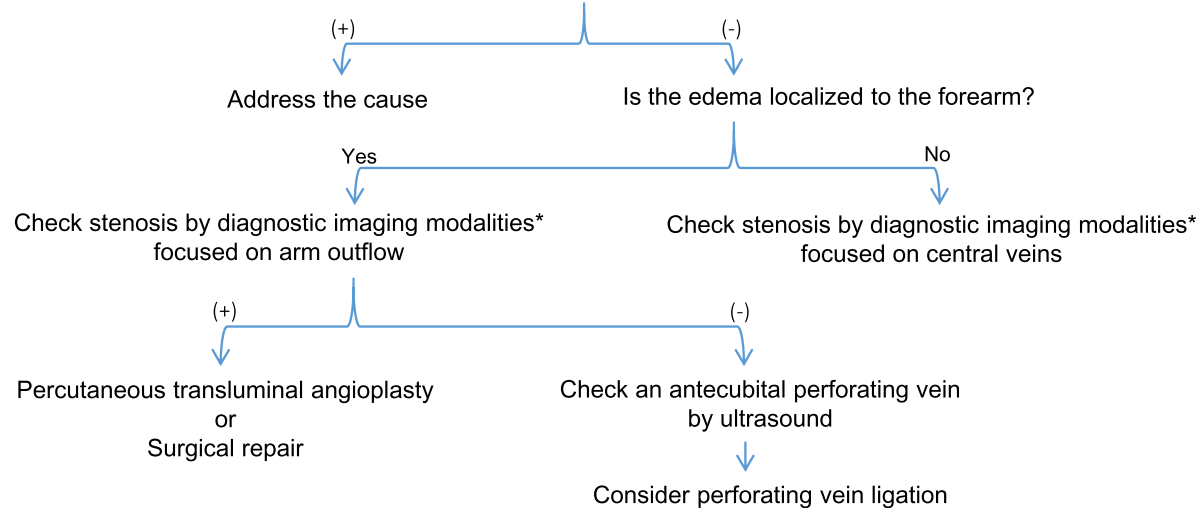

Consider perforating vein ligation

Fig. 5 A flow diagram for the management of AV fistula-related venous hypertension. *One of ultrasound, CT, and angiography or a combination of some of these 
When a practitioner encounters unilateral whole forearm edema without apparent outflow stenosis, it is important to suspect the diagnosis and check both the morphology and flow direction of the antecubital venous bed. Then, perforating vein ligation should be considered if the above-mentioned sonographic sign is obtained, especially in cases of poor forearm superficial veins (Fig. 5).

Unfortunately, we did not perform angiography in the present case, although venous hypertension is usually diagnosed by angiography. However, if the cause is obvious by symptoms, physical exams, and ultrasonography, angiography may not be necessarily required [14], especially when an angiography suite is not readily available, as in our case. Recently, the latest ultrasound equipment was markedly improved in terms of mobility, spatial resolution, and affordability [15]. Indeed, the laptop ultrasound equipment used in our case has highlevel portability, which enabled a prompt diagnosis during the initial outpatient visit. In addition, its spatial resolution was superior at least compared with our 64slice CT system. Portable ultrasound is not only useful for preoperative vascular mapping for AV fistula [16] and procedural guidance for difficult vascular access cannulation [17], but is also effective for the diagnosis of access trouble, as demonstrated in our patient.

\section{Conclusions}

Retrograde outflow through a perforating vein can be a cause of venous hypertension in a patient with an AV fistula created using the proximal radial artery. Close sonographic examination of antecubital vessels should be done if a practitioner encounters unilateral whole forearm edema without apparent proximal outflow stenosis, especially when superficial forearm veins are exhausted.

\section{Additional file}

Additional file 1: Ultrasound image of the perforating vein. Note that the radial artery looks as if it is continuous with the perforating vein in the still longitudinal image due to a slice-thickness artifact. (MP4 $17359 \mathrm{~kb})$

\section{Abbreviations}

$A V$, arteriovenous

\section{Acknowledgements}

None.

\section{Authors' contributions}

TK performed the ultrasound examination and prepared the manuscript. KY and $\mathrm{MO}$ conducted the operation. NI helped to draft the manuscript. All authors read and approved the final manuscript.

\section{Competing interests}

The authors declare that they have no competing interests.

\section{Consent for publication}

Written informed consent was obtained from the patient for the publication of this case report and any accompanying images.

\section{Author details}

${ }^{1}$ Department of Nephrology, Kyoto City Hospital, 1-2, Higashi-takada-cho, Mibu, Nakagyo-ku, Kyoto 604-8845, Japan. ²Okamura Clinic, 24-8, Azeramachi, Imazato, Nagaokakyo, Kyoto 617-0814, Japan.

Received: 26 March 2016 Accepted: 21 July 2016

Published online: 03 October 2016

\section{References}

1. Kukita K, Ohira S, Amano I, Naito H, Azuma N, Ikeda K, et al. 2011 update Japanese society for dialysis therapy guidelines of vascular access construction and repair for chronic hemodialysis. Ther Apher Dial. 2015;19 Suppl 1:1-39.

2. Haruguchi H. Diagnostic methods for vascular access. Tokyo: Chugai Igakusha; 2012. p. 207. Japanese.

3. Kamata T, Ochiai M, Kadoya Y, Tomita M, lehara N. A case of subclavianbrachiocephalic venous junction stenosis diagnosed by a supraclavicular ultrasound. J Jpn Soc Dial Ther. 2013:46:487-91. Japanese.

4. Gracz KC, Ing TS, Soung LS, Armbruster KF, Seim SK, Merkel FK. Proximal forearm fistula for maintenance hemodialysis. Kidney Int. 1977;11:71-5.

5. Palmes D, Kebschull L, Schaefer RM, Pelster F, Konner K. Perforating vein fistula is superior to forearm fistula in elderly haemodialysis patients with diabetes and arterial hypertension. Nephrol Dial Transplant. 2011;26:3309-14.

6. Lomonte C, Basile C. On the phenomenology of the perforating vein of the elbow. Semin Dial. 2009;22:300-3

7. Bruns SD, Jennings WC. Proximal radial artery as inflow site for native arteriovenous fistula. J Am Coll Surg. 2003;197:58-63.

8. Moini M, Williams GM, Pourabbasi MS, Rasouli MR, Tarighi P, Mardanloo A, et al. Side-to-side arteriovenous fistula at the elbow with perforating vein ligation. J Vasc Surg. 2008:47:1274-8.

9. Scholz H. Arteriovenous access surgery. New York: Springer; 2015. p. 135.

10. Padberg Jr FT, Calligaro KD, Sidawy AN. Complications of arteriovenous hemodialysis access: recognition and management. J Vasc Surg. 2008:48:55s-80s.

11. Konner K, Nonnast-Daniel B, Ritz E. The arteriovenous fistula. J Am Soc Nephrol. 2003;14:1669-80.

12. Ten Berge MG, Yo TI, Kerver A, de Smet AA, Kleinrensink GJ. Perforating veins: an anatomical approach to arteriovenous fistula performance in the forearm. Eur J Vasc Endovasc Surg. 2011:42:103-6.

13. Beathard GA, Settle SM, Shields MW. Salvage of the nonfunctioning arteriovenous fistula. Am J Kidney Dis. 1999:33:910-6.

14. Haruguchi H. Diagnostic methods for vascular access. Tokyo: Chugai lgakusha; 2012. p. 208. Japanese.

15. Shafie S, Dickman E. Equipment, image archiving, and billing. In: Soni NJ, Artfield R, Kory P, editors. Point-of-care ultrasound. Philadelphia: Elsevier Saunders; 2015. p. 374-8

16. Ferring $M$, Claridge $M$, Smith SA, Wilmink T. Routine preoperative vascular ultrasound improves patency and use of arteriovenous fistulas for hemodialysis: a randomized trial. Clin J Am Soc Nephrol. 2010;5:2236-44.

17. Kamata T, Tomita M, lehara N. Ultrasound-guided cannulation of hemodialysis access. Ren Replace Ther. 2016;2:7. doi:10.1186/s41100-0160019-1.

Submit your next manuscript to BioMed Central and we will help you at every step:

- We accept pre-submission inquiries

- Our selector tool helps you to find the most relevant journal

- We provide round the clock customer support

- Convenient online submission

- Thorough peer review

- Inclusion in PubMed and all major indexing services

- Maximum visibility for your research 